International Journal of Advanced Astronomy, 9(1) (2021) 24-25
International Journal of Advanced Astronomy
SPC
Website: www.sciencepubco.com/index.php/IJAA
Research paper

\title{
'Oumuamua: A Mysterious Visitor From Deep Space
}

\author{
M. Khalil ${ }^{1 *}$, M. Said ${ }^{1}$, H. Osman ${ }^{1}$, N. Younis ${ }^{2}$, N. Khaled $^{1}$, Y. Amr ${ }^{1}$, N.A. Mohamed ${ }^{1}$, A. Ibrahim ${ }^{1}$ \\ ${ }^{1}$ Faculty of Engineering, October University for Modern Sciences and Arts (MSA), Giza, Egypt \\ ${ }^{2}$ Department of English and American studies, University of Vienna, Austria \\ *Corresponding authorE-mail: mkibrahim@msa.eun.eg
}

\begin{abstract}
The mysterious visitor 'Oumuamua is the first interstellar object ever seen in our solar system. It was discovered by the Pan-STARRS telescope on 19 October 2017. A short overview of 'Oumuamua is presented in this paper. Moreover, we present this work to spotlight on the fact that, there are trillions of such interstellar objects that could visit our solar system.
\end{abstract}

Keywords: 11/2017 U1; 'Ouтиатиa; Interstellar Objects; Pan-STARRS1 Telescope.

\section{Introduction}

In 2017, the Pan-STARRS1 telescope in Hawaii discovered 1I/2017 U1 ('Oumuamua), the first known interstellar visitor coming from outside the solar system $[4,13]$. The name comes from Hawaiian 'Oumuamua 'scout' and reflects the way this object is like a scout or messenger sent from the distant past to reach out to humanity [7].

Firstly, scientists thought the rapidly moving object whose speed was 87.3 kilometers per second $(94,800 \mathrm{~km} / \mathrm{h})$ was a comet or an asteroid that had originated in our solar system. But based on the collected data about its orbit, they realized that it came from the interstellar space. Multiple telescopes focused on this strange rapidly moving object for several nights to determine what it was before it moved out of sights of telescopes $[4,6]$. Scientists believe that 'Oumuamua could have been traveling through the Milky Way galaxy, for hundreds of millions of years, without being attached to any star system, before reaching our solar system [6]. This visitor could carry secrets about how other solar systems have formed. It was a rapidly rotating cigar-shaped object at a size between 100 and 1,000 $\mathrm{m}$ that changed in brightness quite dramatically [4].

\section{The dazzling nature of 'Oumuamua}

Some astronomers identified 'Oumuamua as a comet due to its shiny surface and its highly eccentric orbit but 'Oumuamua showed a strange nature for a comet as it showed no tail as a starter, which in turn did baffle scientists. It caused the initial classification to be considered as an asteroid due to the lack of coma around it [12], but due its visibility being 10x reflective than any observed comets that resides in our solar systems mainly due its infrared radiating nature; its observation was updated to be long period comet with no coma or tail $[1,2,3]$. Its path is not as generic as past comets or asteroids, as it was found that it's not gravitationally bound to the solar system. Its trajectory is hyperbolic, meaning it will keep slipping through our solar system and never revisit. Revealing just how little we know about planetary system beyond our own. On the other hand, some scientists $[5,14]$ have suggested that 'Oumuamua is neither an asteroid nor a comet. It is an irregularly shaped cosmic iceberg.

\section{Chasing 'Oumuamua: what we don't know about 'Oumuamua}

When 'Oumuamua was discovered, scientists estimated that there could be about a hundred trillion interstellar bodies like 'Oumuamua. 2I/Borisov is the second interstellar visitor that has been discovered, but Borisov was definitely a comet with a coma and a tail [10]. Now 'Oumuamua is long gone and is not observable in any way. It is now somewhere between the orbits of Saturn and Neptune and on its way to Interstellar Space in the late 2030s. Scientists believe that there are some unknown features about 'Oumuamua. Several question proposed by NASA $[9,11]$ need to be answered. These questions are as follows:

- What does it look like?

- What is it made of?

- Where did it come from?

- What is it doing now? 


\section{Conclusion and recommendations}

In this work, we present a detailed overview of the mysterious interstellar visitor 'Oumuamua (1I/2017 U1) highlighting its baffling nature to its peer and the importance of its study. The authors encourage and motivate research committees around the globe to study the gathered data and look for visitors in our solar systems as 1I/2017 U1 in order to find answers to the questions in section 3 and to be ready for the upcoming interstellar that may pass through our solar system.

\section{References}

[1] Khalil, M., Said, M., Osman, H., Ahmed, B., Ahmed, D., Younis, N., Maher, B., Osama, M. and Ashmawy, M., Big data in astronomy: from evolution to revolution, International Journal of Advanced Astronomy, 7 (1) (2019) 11-14 https://doi.org/10.14419/ijaa.v7i1.18029.

[2] Khalil, M., Said, M., Osman, H., Younis, N., Khaled N., Amr, Y., Mohamed, N.A., Ibrahim, A., 2020. Dark comets: the cosmic catastrophic threat to earth, 8 (1) (2020) 27-28 https://doi.org/10.14419/ijaa.v8i1.30740.

[3] Khalil, M., Said, M., Osman, H., Younis, N., Khaled N., Amr, Y., Mohamed, N.A., Ibrahim, A., 2020. The Dazzling Comet C/2020 F3 (NEOWISE): The Comet of The Century, 8 (2) (2020) 35-36. https://doi.org/10.14419/ijaa.v8i2.31149.

[4] Meech, K.J., Weryk, R., Micheli, M., Kleyna, J.T., Hainaut, O.R., Jedicke, R., Wainscoat, R.J., Chambers, K.C., Keane, J.V., Petric, A. and Denneau, L., 2017. A brief visit from a red and extremely elongated interstellar asteroid. Nature, 552(7685), pp.378-381. https://doi.org/10.1038/nature25020.

[5] Seligman, D. and Laughlin, G., 2020. Evidence that 1I/2017 U1 ('Oumuamua) was Composed of Molecular Hydrogen Ice. The Astrophysical Journal Letters, 896(1), p.L8. https://doi.org/10.3847/2041-8213/ab963f.

[6] Sugiura, K., 2020. Application: Extremely Elongated Shape of 1I/`Oumuamua. In Development of a Numerical Simulation Method for Rocky Body Impacts and Theoretical Analysis of Asteroidal Shapes (pp. 105-123). Springer, Singapore. https://doi.org/10.1007/978-981-15-3722-6_5.

[7] Astronomy now: 'Oumuamua was a unique object. Now astronomers think there could be trillions just like it (2020). https://astronomynow.com/2020/04/14/oumuamua-was-a-unique-object-now-astronomers-think-there-could-be-trillions-just-like-it/.

[8] CNN: Meet 'Oumuamua, the first observed interstellar visitor to our solar system (2017). https://edition.cnn.com/2017/11/20/world/first-interstellarobject-solar-system/index.html.

[9] Earth Sky: 5 things we know - and 5 we don't - about 'Oumuamua (2018). https://earthsky.org/space/5-things-we-know-dont-about-oumuamua

[10] NASA: Chasing 'Oumuamua (2018). .https://www.nasa.gov/feature/jpl/chasing-oumuamua

[11] NASA: What We Know-And Don't Know-About 'Oumuamua. https://solarsystem.nasa.gov/news/473/what-we-knowand-dont-knowabout-oumuamua/\#: :text=All\%20that\%20astronomers\%20have\%20seen,a\%20single\%20point\%20of\%20light.\&text=But\%20besides\%20its\%20elongated $\% 20$ nature, its $\% 20$ exact $\% 20$ appearance $\% 20$ is $\% 20$ unknown.

[12] Physics world: Interstellar object 'Oumuamua is an asteroid, not a comet argues astrophysicist (2018). https://physicsworld.com/a/interstellar-objectoumuamua-is-an-asteroid-not-a-comet-argues-astrophysicist/

[13] SPACE: 'Oumuamua: The First Interstellar Object (2019). https://www.space.com/oumuamua.html.

[14] The New York times: 'Oumuamua: neither comet nor asteroid, but a cosmic iceberg, 2020. https://www.nytimes.com/2020/06/15/science/oumuamuaastronomy-comets.html\#: :text=Now\%20a\%20pair\%20of\%20Yale,a\%20chunk\%20of\%20frozen\%20hydrogen. 